\title{
Symptom Prediction and Mortality Risk Calculation for COVID-19 Using Machine Learning
}

\begin{abstract}
Elham Jamshidi ${ }^{1 \dagger}$, Amirhossein Asgary ${ }^{2 \dagger}$, Nader Tavakoli ${ }^{3 \dagger}$, Alireza Zali ${ }^{1}$, Farzaneh Dastan ${ }^{4}$, Amir Daaee ${ }^{5}$, Mohammadtaghi Badakhshan ${ }^{6}$, Hadi Esmaily ${ }^{4}$, Seyed Hamid Jamaldini ${ }^{7}$, Saeid Safari ${ }^{1}$, Ehsan Bastanhagh ${ }^{8}$, Ali Maher ${ }^{9}$, Amirhesam Babajani ${ }^{10}$, Maryam Mehrazi ${ }^{3}$, Mohammad Ali Sendani Kashi ${ }^{11}$, Masoud Jamshidi ${ }^{12}$, Mohammad Hassan Sendani ${ }^{13}$, Sahand Jamal Rahi ${ }^{14 *}$ and Nahal Mansouri ${ }^{15,16,17 *}$
\end{abstract}

OPEN ACCESS

Edited by:

Hong Qin,

University of Tennessee at

Chattanooga, United States

Reviewed by:

Ziwei Ma,

University of Tennessee at

Chattanooga, United States

Feng Liu,

Stevens Institute of Technology,

United States

*Correspondence:

Nahal Mansour

nahal.mansouri@chuv.ch

Sahand Jamal Rahi

sahand.rahi@epfl.ch

tThese authors have contributed equally to this work

Specialty section:

This article was submitted to Medicine and Public Health,

a section of the journal

Frontiers in Artificial Intelligence

Received: 27 February 2021

Accepted: 14 May 2021

Published: 22 June 2021

Citation:

Jamshidi E, Asgary A, Tavakoli N,

Zali A, Dastan F, Daaee A,

Badakhshan M, Esmaily $H$,

Jamaldini SH, Safari S, Bastanhagh E,

Maher A, Babajani A, Mehrazi M,

Sendani Kashi MA, Jamshidi $M$,

Sendani MH, Rahi SJ and Mansouri N

(2021) Symptom Prediction and Mortality Risk Calculation for COVID-

19 Using Machine Learning.

Front. Artif. Intell. 4:673527.

doi: 10.3389/frai.2021.673527
${ }^{1}$ Functional Neurosurgery Research Center, Shohada Tajrish Comprehensive Neurosurgical Center of Excellence, Shahid Beheshti University of Medical Sciences, Tehran, Iran, ${ }^{2}$ Department of Biotechnology, College of Sciences, University of Tehran, Tehran, Iran, ${ }^{3}$ Trauma and Injury Research Center, Iran University of Medical Sciences, Tehran, Iran, ${ }^{4}$ Department of Clinical Pharmacy, School of Pharmacy, Shahid Beheshti University of Medical Sciences, Tehran, Iran, ${ }^{5}$ School of Mechanical Engineering, Sharif University of Technology, Tehran, Iran, ${ }^{6}$ School of Electrical and Computer Engineering, Engineering Faculty, University of Tehran, Tehran, Iran, ${ }^{7}$ Department of Genetic, Faculty of Advanced Science and Technology, Tehran Medical Sciences, Islamic Azad University, Tehran, Iran, ${ }^{8}$ Department of Anesthesiology, Tehran University of Medical Sciences, Tehran, Iran, ${ }^{9}$ School of Management and Medical Education, Shahid Beheshti University of Medical Sciences, Tehran, Iran, ${ }^{10}$ Department of Pharmacology, School of Medicine, Shahid Beheshti University of Medical Sciences, Tehran, Iran, ${ }^{11}$ Department of Business Management, Faculty of Management, University of Tehran, Tehran, Iran, ${ }^{12}$ Department of Exercise Physiology, Tehran University, Tehran, Iran, ${ }^{13}$ Department of Computer Engineering, Sharif University of Technology, Tehran, Iran, ${ }^{14}$ Laboratory of the Physics of Biological Systems, Institute of Physics, École Polytechnique Fédérale de Lausanne (EPFL), Lausanne, Switzerland, ${ }^{15}$ Division of Pulmonary Medicine, Department of Medicine, Lausanne University Hospital (CHUV), University of Lausanne (UNIL), Lausanne, Switzerland, ${ }^{16}$ Swiss Institute for Experimental Cancer Research (ISREC), School of Life Sciences, École Polytechnique Fédérale de Lausanne (EPFL), Lausanne, Switzerland, ${ }^{17}$ Research Group on Artificial Intelligence in Pulmonary Medicine, Division of Pulmonary Medicine, Lausanne University Hospital (CHUV), Lausanne, Switzerland

Background: Early prediction of symptoms and mortality risks for COVID-19 patients would improve healthcare outcomes, allow for the appropriate distribution of healthcare resources, reduce healthcare costs, aid in vaccine prioritization and self-isolation strategies, and thus reduce the prevalence of the disease. Such publicly accessible prediction models are lacking, however.

Methods: Based on a comprehensive evaluation of existing machine learning (ML) methods, we created two models based solely on the age, gender, and medical histories of 23,749 hospital-confirmed COVID-19 patients from February to September 2020: a symptom prediction model (SPM) and a mortality prediction model (MPM). The SPM predicts 12 symptom groups for each patient: respiratory distress, consciousness disorders, chest pain, paresis or paralysis, cough, fever or chill, gastrointestinal symptoms, sore throat, headache, vertigo, loss of smell or taste, and muscular pain or fatigue. The MPM predicts the death of COVID-19-positive individuals.

Results: The SPM yielded ROC-AUCs of 0.53-0.78 for symptoms. The most accurate prediction was for consciousness disorders at a sensitivity of $74 \%$ and a specificity of $70 \%$.

Abbreviations: AI, artificial intelligence; COVID-19, coronavirus disease of 2019; intensive care unit, IQR; interquartile range, KS; Kolmogorov-Smirnov, LR; logistic regression, ML; machine learning, RF; random forest, RDW; red blood cell, distribution width; ROC, receiver operating characteristic; HIS, hospital information system. 
2,440 deaths were observed in the study population. MPM had a ROC-AUC of 0.79 and could predict mortality with a sensitivity of $75 \%$ and a specificity of $70 \%$. About $90 \%$ of deaths occurred in the top 21 percentile of risk groups. To allow patients and clinicians to use these models easily, we created a freely accessible online interface at www. aicovid.net.

Conclusion: The ML models predict COVID-19-related symptoms and mortality using information that is readily available to patients as well as clinicians. Thus, both can rapidly estimate the severity of the disease, allowing shared and better healthcare decisions with regard to hospitalization, self-isolation strategy, and COVID-19 vaccine prioritization in the coming months.

\section{Keywords: COVID-19, artificial intelligence, machine learning, symptom, mortality}

\section{INTRODUCTION}

The COVID-19 pandemic of the 2019 novel coronavirus (SARSCoV-2) started in December 2019 and is spreading rapidly, with approximately 62.5 million confirmed cases and 1.5 million deaths by the end of November 2020 (WHO, 2020).

The severity of the disease varies widely between different patients, ranging from no symptoms to a mild flu-like illness, to severe respiratory symptoms, and to multi-organ failure leading to death. Among the symptoms, fever, cough, and respiratory distress are more prevalent than symptoms such as consciousness disorders and loss of smell and taste (Tabata et al., 2020; Jamshidi et al., 2021b). In general, complications are common among elderly patients and those with pre-existing conditions. The intensive care unit (ICU) admission rate is substantially higher for these groups (Abate et al., 2020; Jamshidi et al., 2021a).

The Center for Disease Control (CDC) and the World Health Organization (WHO) consider the identification of individuals at higher risk a top priority. This identification could be used for numerous solutions to moderate the consequences of the pandemic for the most vulnerable (CDC COVID-19 Response Team, 2020) as well as minimize the presence of actively ill patients in society.

This requires the prediction of the symptoms and mortality risk for infected individuals. While symptom prediction models exist for cancer, no such models have been designed for COVID19 (Levitsky et al., 2019; Goecks et al., 2020). To make rapid, evidence-based decisions possible, they will ideally be based on readily available patient information, i.e., demographic attributes and past medical history (PMH) as opposed to costly laboratory tests. Early decision-making is critical for timely triage and clinical management of patients. For instance, clinical and laboratory data can only be assessed after presenting the individual to a health care center, increasing the risk of unnecessary exposures to the virus and increasing costs (Sun et al., 2020). These parameters are not available immediately and are partly subject to human error. Also, factors like genetic predisposition may increase the models' accuracy but are not broadly available.
With the growth of big data in healthcare and the introduction of electronic health records, artificial intelligence (AI) algorithms can be integrated into hospital IT systems and have shown promise as computer-aided diagnosis and prognostic tools. In the era of COVID-19, AI has played an essential role in the early diagnosis of infection, the prognosis of hospitalized patients, contact tracing for spread control, and drug discovery (Lalmuanawma et al., 2020). AI methods can have a higher accuracy over classical statistical analyses.

In contrast to the few previously available COVID-19 risk scales, our mortality prediction model uses a selection of variables that are in principle accessible to all patients and thus can be used immediately after diagnosis (Assaf et al., 2020; Pan et al., 2020). This model not only has a significant benefit in early decision making in the hospital setting, but because it does not require clinicians or laboratories, it can serve as a triage tool for patients in an outpatient setting, in telemedicine, or as a self-assessment tool. For example, decisions on outpatient vs. inpatient care can be made remotely by estimating the most probable symptoms and severity risks. This lessens the strain on health care resources, unnecessary costs, and unwanted exposures to infected patients.

Here, we implemented $2 \mathrm{ML}$ methods to predict the symptoms and the mortality of patients with COVID-19. Overall, 23,749 patients were included in the study. The predictors used for the models were age, sex, and PMH of the patients. Both of these models achieved predictions with high accuracy. To our knowledge, this is one of the largest datasets of COVID-19 cases and is the only study that uses patient-available data for the prediction of COVID-19 symptoms and mortality. Furthermore, this study is the most extensive study for mortality prediction for COVID-19 using ML-based on any set of predictors (An et al., 2020; Gao et al., 2020; Vaid et al., 2020; Yadaw et al., 2020).

We also created an online calculator where each individual can predict their COVID-19 related symptoms and risk (www. aicovid.net).

For a standardized representation of the methodology and results of this analysis an adapted version of the Transparent Reporting of Multivariable Prediction Model for Individual Prognosis or Diagnosis (TRIPOD) guideline was followed (Collins et al., 2015). 
TABLE 1 | List of predictors. Predictor variables for mortality risk and symptom prediction of COVID-19.

\begin{tabular}{|c|c|c|}
\hline Category & Variable & Description \\
\hline \multirow[t]{2}{*}{ Demographic } & Age & In years \\
\hline & Sex & Male or female \\
\hline \multirow[t]{13}{*}{$\begin{array}{l}\text { Past/Current Medical } \\
\text { Conditions }\end{array}$} & Cancer & $\begin{array}{l}\text { Current chemotherapy, radiotherapy, immunotherapy, bone marrow or stem cell } \\
\text { transplantation }\end{array}$ \\
\hline & Liver disorders & $\begin{array}{l}\text { Chronic hepatitis (type B or C), alcohol-related liver disease, primary biliary cirrhosis, primary } \\
\text { sclerosing cholangitis, hemochromatosis, cirrhosis }\end{array}$ \\
\hline & Blood disorders & $\begin{array}{l}\text { Anemia (iron deficiency, thalassemia minor and major, sickle cell disease), coagulopathies } \\
\text { (hemophilia and platelet disorders) }\end{array}$ \\
\hline & Immune disorders & $\begin{array}{l}\text { Immune deficiency (acquired immunodeficiency syndrome, treatment with steroids and } \\
\text { immune suppressors), autoimmune disease (rheumatoid arthritis, systemic lupus } \\
\text { erythematosus, ankylosing spondylitis, vasculitis). }\end{array}$ \\
\hline & Cardiovascular disease & $\begin{array}{l}\text { Congestive heart failure, cardiovascular events (myocardial infarction, stroke, angina), valvular } \\
\text { heart disease, arrhythmia (e.g. atrial fibrillation) }\end{array}$ \\
\hline & Kidney disorders & Chronic kidney disease (stage 3, 4, and end-stage renal disease) \\
\hline & Respiratory disorders & $\begin{array}{l}\text { Asthma, chronic obstructive pulmonary disease (emphysema and chronic bronchitis), extrinsic } \\
\text { allergic alveolitis, cystic fibrosis, interstitial lung disease, sarcoidosis, bronchiectasis, pulmonary } \\
\text { hypertension }\end{array}$ \\
\hline & Neurological disorders & $\begin{array}{l}\text { Epilepsy, Parkinson's disease, motor neuron disease, cerebral palsy, dementia, multiple } \\
\text { sclerosis }\end{array}$ \\
\hline & Endocrine disorders & $\begin{array}{l}\text { Hyperthyroidism, hypothyroidism, cushing syndrome, pheochromocytoma, thyroiditis, } \\
\text { hyperaldosteronism }\end{array}$ \\
\hline & Diabetes mellitus & $\begin{array}{l}\text { Type } 1 \text { and type } 2 \text { diabetes, maturity onset diabetes of the young, insipidus, gestational } \\
\text { diabetes }\end{array}$ \\
\hline & Hypertension & Primary and secondary \\
\hline & $\begin{array}{l}\text { Psychiatric disorders (removed due to low } \\
\text { prevalence) }\end{array}$ & Bipolar disorder, psychosis, schizophrenia, major depression disorder \\
\hline & $\begin{array}{l}\text { Thrombosis (removed due to low } \\
\text { prevalence) }\end{array}$ & Venous thromboembolism, pulmonary thromboembolism \\
\hline
\end{tabular}

\section{METHODS}

\section{Source of Data and Participants}

In this cohort study, we used the Hospital Information System (HIS) of 74 secondary and tertiary care hospitals across Tehran, Iran. The eligibility criteria were defined as confirmed or suspected SARS-CoV-2 infections of people aged 18-100 years registered in the referred HIS. The final database used to design the models was obtained by aggregating the 74 hospitals' HIS. The study included patients referred to any of the hospitals between February 1, 2020, and September 30, 2020. Patients were followed up through October 2020 until all the registered patients had the specific death or survival outcome needed for the mortality prediction model (MPM). This study was approved by the Iran University of Medical Sciences Ethics Committee.

\section{Outcome}

\section{Symptom Prediction Model}

The patients' symptoms at the time of admission, as recorded in the HIS, were considered as the outputs of the Symptom Prediction Model (SPM). All stated symptoms were clustered in 12 categories to be predicted by the model. The groups are cough, loss of smell or taste, respiratory distress, vertigo, muscular pain or fatigue, sore throat, fever or chill, paresis or paralysis, gastrointestinal problems, headache, chest pain, and consciousness disorders.

\section{Mortality Prediction Model}

Death or survival as per the HIS records was defined as the output of the mortality prediction model (MPM).

\section{Predictors}

The patients' age, sex, and past medical history $(\mathrm{PMH})$, as detailed in Table 1, were used as predictors for both models. The selection of variables as predictors was based on the available recorded data. All these predictors were recorded in the HIS at the time of admission.

\section{Missing Data}

We only included patients with the required data. Due to the absence of missing data, there was no imputation of missing values.

\section{Pre-Processing}

Symptoms and predictor variables from the medical histories with an incidence of less than $0.2 \%$ were removed to reduce noise. This removed past COVID-19 infections, thrombosis, psychiatric disorders, and organ or bone marrow transplantation from the set of predictor variables. The removed symptoms were tachycardia, seizure, nasal congestion, and skin problems.

Sex, $\mathrm{PMH}$, and symptoms were encoded as binary variables. In training and test sets, the only continuous predictor, age, was standardized to zero mean and unit standard deviation. 
TABLE 2 | Patient characteristics and symptoms. Baseline characteristics, symptoms, and death outcomes for COVID-19 patients.

\begin{tabular}{|c|c|}
\hline Variable & Median ( \pm IQR) \\
\hline Age & $52( \pm 29)$ \\
\hline \multicolumn{2}{|l|}{ Categorical/Binary variables } \\
\hline Variable & Count (percent) \\
\hline \multicolumn{2}{|l|}{ Sex } \\
\hline Male & 12,597 (53.04\%) \\
\hline Female & $11,152(46.96 \%)$ \\
\hline Cardiovascular disease & $2,471(10.4 \%)$ \\
\hline Diabetes & $2,068(8.71 \%)$ \\
\hline Hypertension & 2,004 (8.44\%) \\
\hline Respiratory diseases & $546(2.3 \%)$ \\
\hline Cancer & 477 (2.01\%) \\
\hline Kidney disorders & $416(1.75 \%)$ \\
\hline Neurological disorders & $264(1.11 \%)$ \\
\hline Immune disorders & $178(0.75 \%)$ \\
\hline Blood disorders & 152 (0.64\%) \\
\hline Current pregnancy & 139 (0.59\%) \\
\hline Liver disorders & $119(0.5 \%)$ \\
\hline Endocrine disorders & $97(0.41 \%)$ \\
\hline Organ or bone marrow transplant & $29(0.12 \%)$ \\
\hline Mental illnesses & 19 (0.08\%) \\
\hline Thrombosis & 15 (0.06\%) \\
\hline Past COVID-19 infection & $10(0.04 \%)$ \\
\hline \multicolumn{2}{|l|}{ Outcomes } \\
\hline Survived & 21,309 (89.73\%) \\
\hline Dead & 2,440 (10.27\%) \\
\hline \multicolumn{2}{|l|}{ Symptoms } \\
\hline Cough & $11,995(50.51 \%)$ \\
\hline Respiratory distress & $10,342(43.55 \%)$ \\
\hline Muscular pain or fatigue & 9,249 (38.94\%) \\
\hline Fever or chill & 8,553 (36.01\%) \\
\hline Gastrointestinal problems & 2,469 (10.4\%) \\
\hline Headache & $1,120(4.72 \%)$ \\
\hline Chest pain & 745 (3.14\%) \\
\hline Consciousness disorders & $698(2.94 \%)$ \\
\hline Loss of smell or taste & $659(2.77 \%)$ \\
\hline Vertigo & $501(2.11 \%)$ \\
\hline Sore throat & 157 (0.66\%) \\
\hline Paresis or paralysis & $121(0.51 \%)$ \\
\hline
\end{tabular}

\section{Machine Learning Methods}

To ensure generalizability, a 5-fold cross-validation algorithm was employed [Performance evaluation of classification algorithms by $\mathrm{k}$-fold and leave-one-out cross validation, (Wong, 2015). All records were randomly separated into five independent subsets. Four subsets were used as training data, and one subset was retained as a validation set for model testing. The cross-validation process was then iterated four more times, with each of the five subsets being used as validation data exactly once. Subsequently, model performance metrics were evaluated for training and validation groups separately in each model iteration.

By separating deceased and surviving patients separately into five mortality-stratified subsets first and then combining these into the final five subsets, we maintained the same proportion of deceased and surviving patients in each of the final five subsets.

We evaluated several machine learning techniques for both models: Logistic Regression, Random Forest, Artificial Neural
Network (ANN), K-Nearest Neighbors (KNN), Linear Discriminant Analysis (LDA), and Naive Bayes.

We took advantage of the Scikit-learn machine learning library to implement both preprocessing algorithms and models (Garreta and Moncecchi, 2013).

\section{Symptom Prediction Model}

The SPM output predicts symptoms for SARS-CoV-2 positive patients. Since there are 12 symptom groups, we judged the models' overall performance by a single metric, the prevalenceweighted mean of the twelve ROC-AUCs (Mandrekar, 2010), in which the ROC-AUCs were weighted by symptom prevalence.

\section{Mortality Prediction Model}

The MPM calculates the probability of death for SARS-CoV-2 positive patients. Each model's performance was measured in terms of a ROC-AUC.

\section{RESULTS}

\section{Participants}

Baseline characteristics of patients and their symptoms are shown in Table 2. Of all 23,749 confirmed or suspected COVID-19 patients, 2,440 (10.27\%) passed away at the end of the study (see Discussion). A comparison of the characteristics of survived and deceased patients is shown in Table 3. A comparison of the characteristics of patients with and without each symptom is shown in Supplementary Tables S1-S16.

We used statistical hypothesis tests to demonstrate each predictor variable's significance to the model outputs. We employed the F-test (Snedecor, 1957) technique for age, a continuous variable, and the Chi-square (Snedecor, 1957) technique for other categorical variables such as sex and $\mathrm{PMH}$.

\section{Model Specification}

We evaluated six machine learning methods for both the SPM and MPM, which are listed, together with the hyperparameters used in Table 4.

\section{Model Performance Symptom Prediction Model}

The SPM can be considered as 12 separated classifiers; each predicts the occurrence of a specific symptom. While the performance of each sub-classifier can be evaluated separately, the overall performance can be assessed using the prevalence-weighted mean of the ROC-AUCs, since the symptoms have different prevalence. The prevalenceweighted mean ROC-AUC for each method is illustrated in Figure 1. Although the $\mathrm{KNN}$ method provided the highest weighted mean ROC-AUC for the test data, it was the least robust method since its performance varied considerably for different validation folds (note standard deviation bars). The Random Forest method achieved better overall performance and robustness. The weighted mean ROC-AUC value of this method was 0.582 for the test data. 
TABLE 3 | Comparison between survived and deceased patient groups. Comparative evaluation of the characteristics of survived and deceased COVID-19 patients.

Continuous variables

\begin{tabular}{|c|c|c|c|c|}
\hline Variable & $\begin{array}{c}\text { Median } \\
\text { in survivors }( \pm I Q R)\end{array}$ & $\begin{array}{c}\text { Median } \\
\text { in deceased }( \pm I Q R)\end{array}$ & F-test statistics & F-test $p$-value \\
\hline Age & $49( \pm 27)$ & $70( \pm 21)$ & $2,039.47$ & $<0.001$ \\
\hline \multicolumn{5}{|l|}{ Categorical/Binary variables } \\
\hline Variable & Count in survivors (percent in survivors) & Count in deceased (percent in deceased) & Chi2 statistics & Chi2 $p$-value \\
\hline \multicolumn{5}{|c|}{ 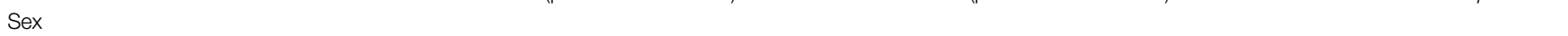 } \\
\hline Male & 11,163 (52.39\%) & $1,434(58.77 \%)$ & 16.82 & $<0.001$ \\
\hline Female & $10,146(47.61 \%)$ & $1,006(41.23 \%)$ & 19 & $<0.001$ \\
\hline Cardiovascular disease & $2,039(9.57 \%)$ & 432 (17.7\%) & 139.29 & $<0.001$ \\
\hline Diabetes & $1,693(7.94 \%)$ & $375(15.37 \%)$ & 138.57 & $<0.001$ \\
\hline Hypertension & $1,676(7.87 \%)$ & $328(13.44 \%)$ & 80.71 & $<0.001$ \\
\hline Respiratory disorders & $462(2.17 \%)$ & $84(3.44 \%)$ & 15.47 & $<0.001$ \\
\hline Cancer & $343(1.61 \%)$ & $134(5.49 \%)$ & 164.28 & $<0.001$ \\
\hline Kidney disorders & 317 (1.49\%) & 99 (4.06\%) & 82.54 & $<0.001$ \\
\hline Neurological disorders & 207 (0.97\%) & 57 (2.34\%) & 36.68 & $<0.001$ \\
\hline Immune disorders & $152(0.71 \%)$ & $26(1.07 \%)$ & 3.62 & 0.057 \\
\hline Blood disorders & $112(0.53 \%)$ & 40 (1.64\%) & 42.43 & $<0.001$ \\
\hline Current pregnancy & $133(0.62 \%)$ & $6(0.25 \%)$ & 5.35 & 0.021 \\
\hline Liver disorders & $101(0.47 \%)$ & $18(0.74 \%)$ & 3.04 & 0.081 \\
\hline Endocrine disorders & $88(0.41 \%)$ & $9(0.37 \%)$ & 0.1 & 0.747 \\
\hline Organ or bone marrow transplant & $25(0.12 \%)$ & $4(0.16 \%)$ & 0.39 & 0.533 \\
\hline Psychiatric disorders & $16(0.08 \%)$ & $3(0.12 \%)$ & 0.63 & 0.428 \\
\hline Thrombosis & $13(0.06 \%)$ & $2(0.08 \%)$ & 0.15 & 0.696 \\
\hline Past COVID-19 infection & $10(0.05 \%)$ & $0(0.0 \%)$ & 1.15 & 0.285 \\
\hline
\end{tabular}

TABLE 4 | Machine learning methods and hyperparameters used.

\section{The Mortality Prediction Model}

\begin{tabular}{|c|c|c|}
\hline Method & Parameter & Value(s) \\
\hline Logistic Regression & $\mathrm{C}$ & 1.0 \\
\hline \multirow[t]{3}{*}{ Random Forest } & Number of trees & 500 \\
\hline & Min. Number of samples at a leaf node & $\% 0.1$ of all samples \\
\hline & Criterion & Gini \\
\hline \multirow[t]{3}{*}{ Artificial Neural Networks } & Number of layers & 3 \\
\hline & Output space dimensionality for each layer & $32,16,1$ \\
\hline & Activation function for each layer & Tanh, tanh, sigmoid \\
\hline \multirow[t]{2}{*}{ K-Nearest Neighbors } & $\mathrm{K}$ & 10 \\
\hline & Weight function & Distance \\
\hline Linear Discriminant Analysis & Solver & SVD \\
\hline Naive Bayes & Interval size of age categories & 0.1 \\
\hline \multicolumn{3}{|c|}{ The Symptom Prediction Model } \\
\hline Method & Parameter & Value \\
\hline Logistic Regression & C & 1.0 \\
\hline \multirow[t]{3}{*}{ Random Forest } & Number of trees & 200 \\
\hline & Min. Number of samples at a leaf node & $\% 0.1$ of all samples \\
\hline & Criterion & Gini \\
\hline \multirow[t]{3}{*}{ Artificial Neural Network } & Number of layers & 4 \\
\hline & Output space's dimensionality for each layer & $32,32,32,12$ \\
\hline & Activation function for each layer & Tanh, tanh, tanh, tanh, sigmoid \\
\hline \multirow[t]{2}{*}{ K-Nearest Neighbors } & $\mathrm{K}$ & 5 \\
\hline & Weight function & Distance \\
\hline Linear Discriminant Analysis & Solver & SVD \\
\hline Naive Bayes & Interval size of age categories & 0.1 \\
\hline
\end{tabular}

Moreover, the performance of the SPM can be evaluated for each symptom separately. The ROC-AUC values for predicting consciousness disorder, paresis or paralysis, and chest pain were $0.785,0.729$, and 0.686 , respectively. Also, at a specificity of $70 \%$, the sensitivities were $73 \%, 50 \%$, and $53 \%$, respectively.

As shown in Figure 1, the random forest model with a mean ROC-AUC of 0.8 and 0.79 has the highest efficiency in the 


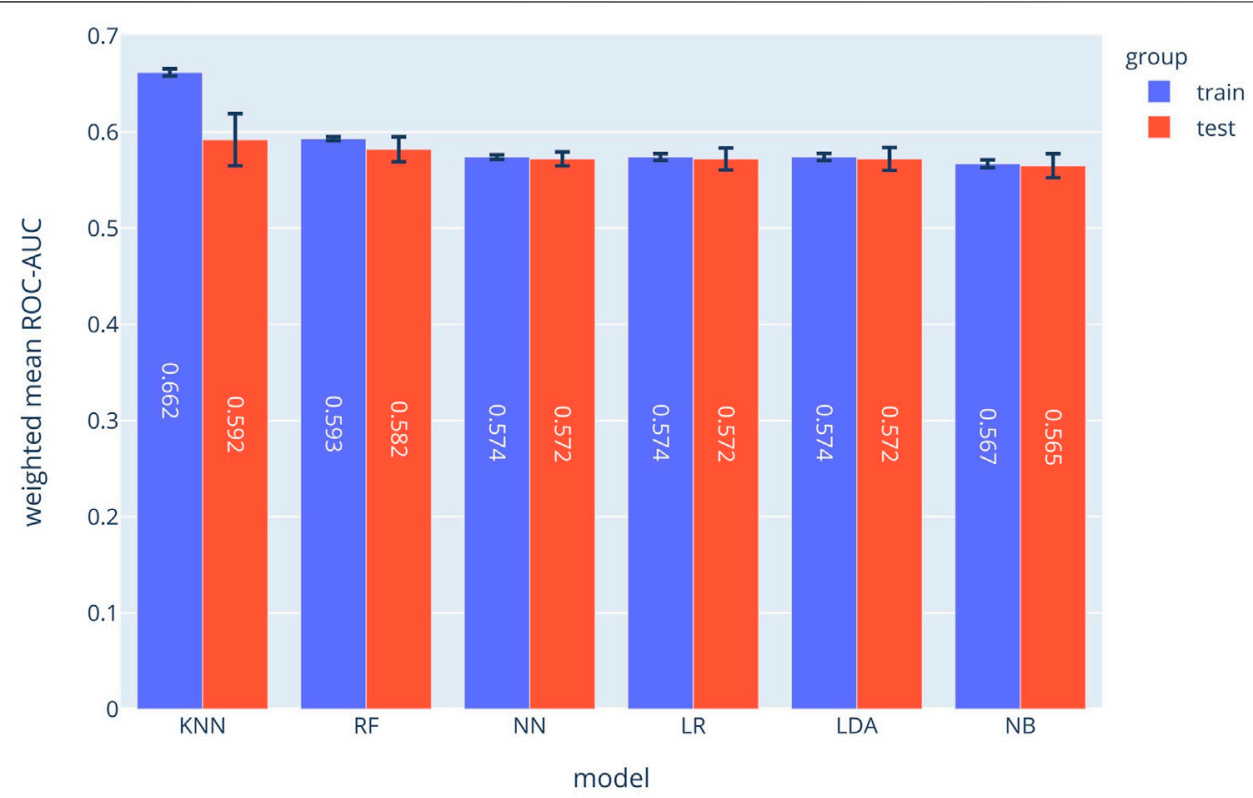

FIGURE 1 | Prevalence-weighted means ROC-AUCs for different ML models. The models were used to implement the Symptom Prediction Model (SPM). Error bars denote the standard deviation over different cross-validation folds.

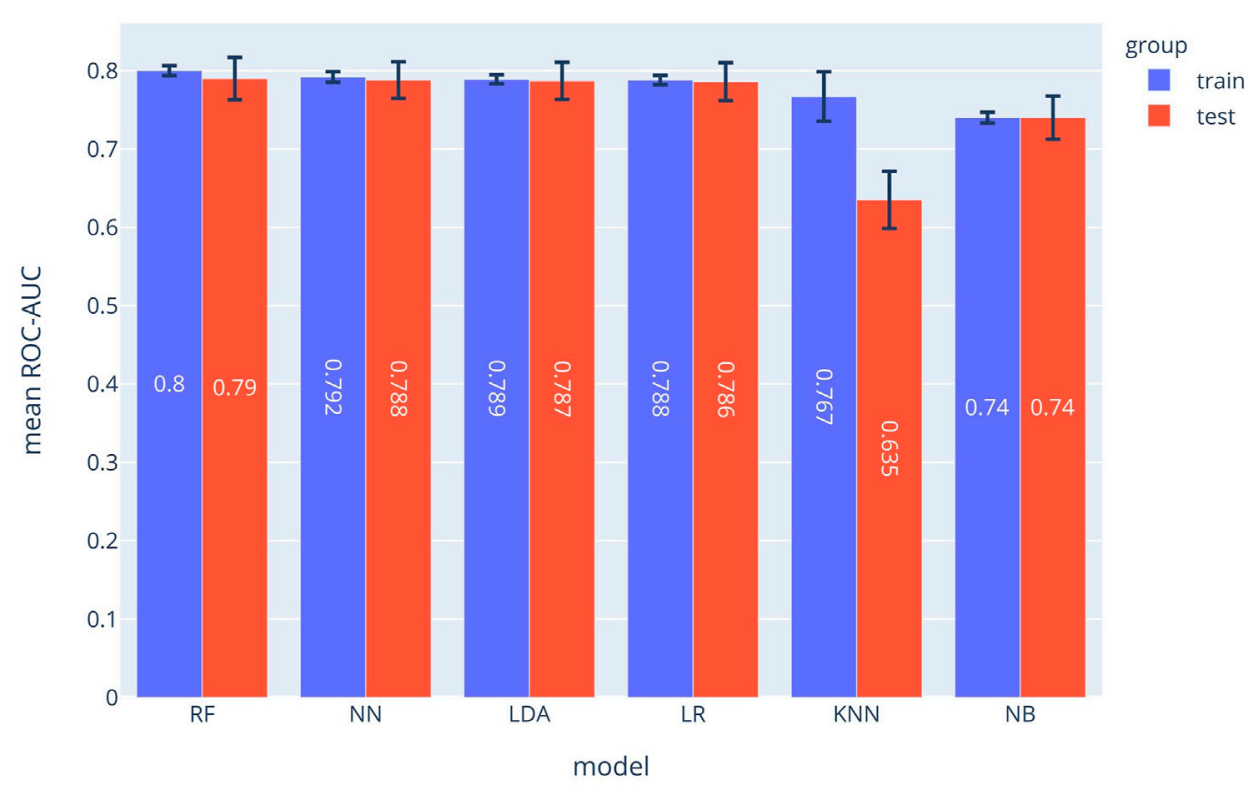

FIGURE 2 | ROC-AUCs of different ML models which were used to implement the MPM. The Random Forest (RF) model outperformed the other approaches.

training and the validation groups, respectively, followed by the Neural Network and LDA. In the symptom prediction model, the ROC-AUC values of all models in addition to the weighted average of ROC-AUC of different ML methods for each symptom are shown in Supplementary Figure S1. Supplementary Figure S2 delineates each method's performance for all symptoms as a Radar chart.
Based on the ROC diagram and the information from the database, the other performance metrics of the other models were identified. In addition to the ROC-AUC of the risk prediction model, we calculated the sensitivity and the negative and positive predictive value (NPV and PPV respectively) for each model. The detailed results of all six algorithms for both MPM and SPM are shown in Supplementary Tables S17 and S18. 
The calibration plot of the RF implementation for each symptom predictor (sub-classifier) is depicted in Supplementary Figure S3 which shows the calibration plot of the RF implementation of each symptom.

\section{Mortality Prediction Model}

The ROC-AUC values for each method are depicted in Figure 2. In the MPM classifier, the Random Forest method outperformed the other methods just as for the SPM. The achieved ROC-AUC value was 0.79 for the test data.

Supplementary Figure S4 shows ROC diagrams representing the true-positive rates vs. false-positive rates for each method used to implement the MPM. The calibration plot of the RF model is depicted in Supplementary Figure S5. Calibration indications such as Mean Calibration Error are also shown in the Supplementary Figure S5 for different methods.

\section{Model Input-Output Correlations}

We used the Chi-square test and the F-test to evaluate the extent to which PMH, sex, or age predict the outputs of the SPM and MPM. The larger the values of these test values are for each predictor variable, the more the predictor variable is predictive of the output of the models. For categorical predictor variables (i.e., PMH and sex), the Chi-square hypothesis test was used. To evaluate the predictive value of a categorical variable, we examined whether it was more common in patients who died (MPM) or in patients with a particular symptom (SPM). For the only continuous variable (age), we used the F-test. To find the impact of age, we examined if the age median was higher in dead patients (MPM) or patients with a particular symptom (SPM).

For the SPM model, Supplementary Figure S6 shows how each factor in the PMH was correlated with each symptom using the Chi-square test. For example, patients with diabetes or cardiovascular disease were more likely to have consciousness disorders and chest pain in case of infection with COVID-19. The effect of age on each symptom is shown in Supplementary Figure S7 using the F-test. Older patients were more likely to develop symptoms such as respiratory distress and consciousness disorder but also less likely to develop symptoms such as muscular pain or fatigue.

In addition, for the MPM, the impact of each PMH on death is shown in Supplementary Figure S8. In our analysis, cancer, cardiovascular disease, and diabetes have the greatest effects on the risk of death in patients with Covid 19; on the other hand, pregnancy or being female decreased the chances of death. The F-test statistic of age in the MPM model is 2,039.47, which explains the increase in mortality risk from aging.

DeLong's test shows the statistically significant difference between AUCs of models. The DeLong tests for the MPM and SPM predictions are shown through Supplementary Figures S9-S21.

\section{Validation of the Model for Each Mortality Peak}

For additional validation of our model, we evaluated the performance of the final random forest for MPM during the periods with the highest rate of mortality. The data corresponding to each available mortality peak (april, February, and September 2020) was selected from the validation dataset of each model, and the outcome (recovery or death of the patient) during each period was predicted by the model and shown as a ROC diagram (Figure 3). Despite the variation of the AUC in the mortality peaks, the weighted average of the AUC values corresponding to each period was approximately equal to the average model yield for the entire data. We can conclude that the model continues to perform equally well during each mortality peak. The cause of the high yield in april could be explained by the large number of available samples which would allow the algorithm to learn more accurately.

\section{DISCUSSION}

Our objective in this study was to develop $2 \mathrm{ML}$ models to predict the mortality and symptoms of COVID-19-positive patients among the general population using age, gender, and comorbidities alone. These models can guide the design of measures to combat the COVID-19 pandemic. The prediction of vulnerability using the models allows people in different risk groups to take appropriate actions if they contract COVID-19. For example, people who fall into the low-risk group can start isolation sooner when the predicted symptoms appear and refer to a hospital only if the symptoms persist. As a result, the risk of disease spread and the pressure on the health care system from unnecessary hospital visits, costs, and psychological and physical stress to the medical staff could be reduced (Emanuel et al., 2020). In contrast, people who are predicted to be at higher risk are recommended to seek medical care immediately. Predictions can speed up the treatment process and ultimately decrease mortality.

Our study has shown that multiple symptoms have strong correlations with different medical history factors. Symptoms can be either amplified or attenuated by health backgrounds; for instance, hypertension, diabetes, and respiratory and neurological disorders increased the chances of loss of smell or taste; however, pregnancy, cancer, higher age, cardiovascular disease, and liver, immune system, blood, and kidney disorders have attenuated the appearance of this symptom.

Due to the complexity of the COVID-19 pathogenesis, many clinical studies revealed contradictory results, for example, the effectiveness or ineffectiveness of remdisivir (Beigel et al., 2020; Goldman et al., 2020; Wang et al., 2020). We hypothesize that the imbalance of mortality risks between the intervention and control groups could have been a problem in these studies. With the help of our model, such problems could be partially solved by equalizing the mortality baseline in different clinical groups.

Our AI models can also be beneficial for COVID-19 vaccine testing and prioritization strategies. The limited number of approved vaccines in the first months of the vaccination process and the potential shortages make vaccine prioritization inevitable. This prioritization would be more important for developing countries that do not have the resources to pre- 


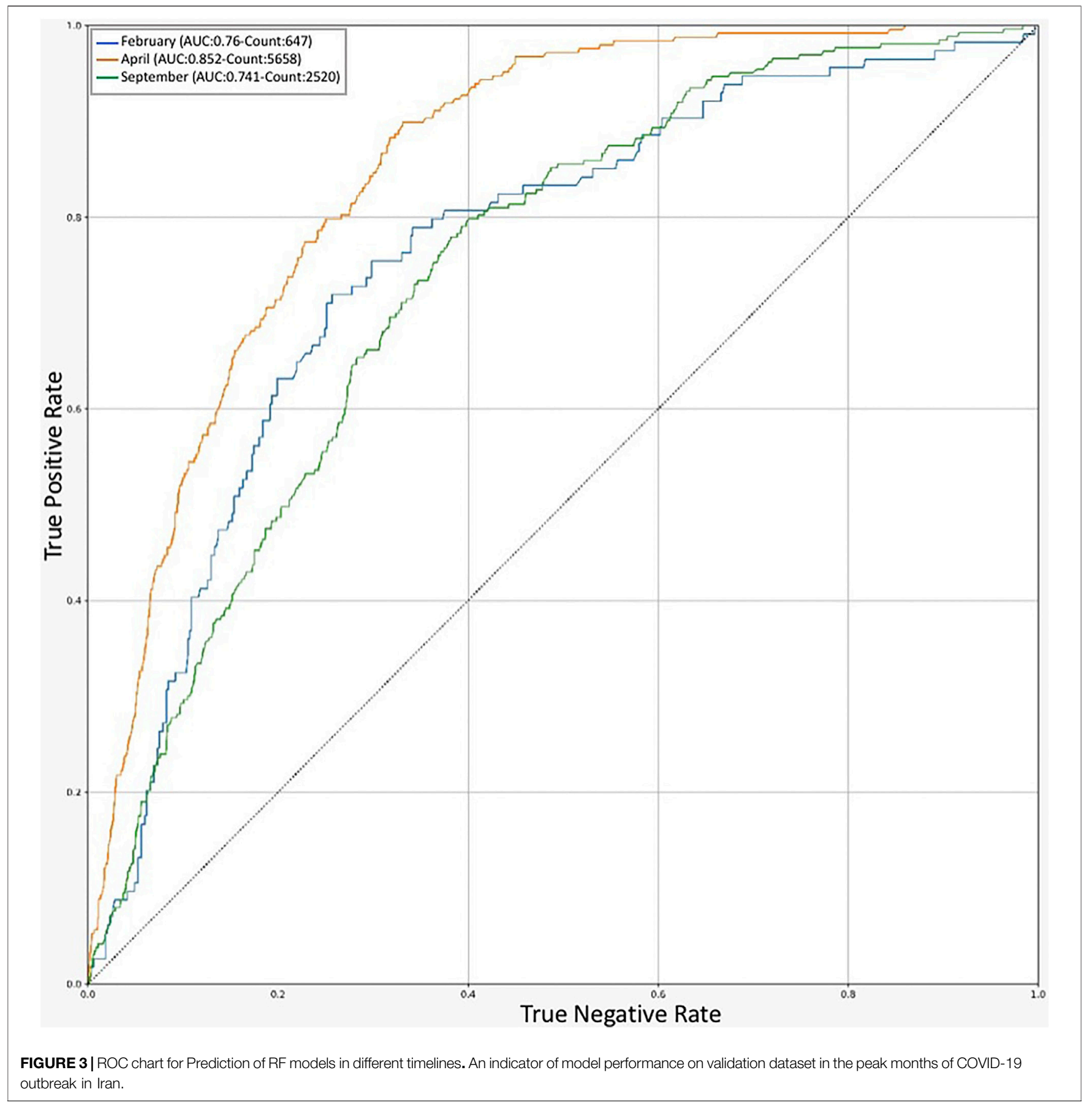

order vaccines from multiple companies (Persad et al., 2020). Having a mortality prediction tool for each individual could be a valuable tool for governments to decide on vaccines' allocation.

\section{Limitations}

Since our dataset was collected by the HIS, it did not contain COVID-19 patients that did not refer to a hospital or had no major symptoms to be identified as infected. This could explain the high mortality rate in our and other studies (Fumagalli et al.,
2020; Gue et al., 2020; Yadaw et al., 2020). However, for a systematic study with few confounding variables, uniform data collection is essential, which can only be realistically ensured with hospital data.

Also, other variables such as the viral load may be important but are difficult to measure and are not readily available. We opted for easily accessible predictor variables to allow the widespread use of the models.

One way to improve the models is to subgroup-specific factors in the medical history or specific symptoms further. The main 
reason for grouping factors and symptoms was the low prevalence of certain subsets in the dataset.

In conclusion, we evaluated 15 parameters (Table 1) for predicting the symptoms and the mortality risk of COVID-19 patients. The ML models trained in this study could help people quickly determine their mortality risk and the probable symptoms of the infection. These tools could aid patients, physicians, and governments with informed and shared decision-making.

\section{DATA AVAILABILITY STATEMENT}

The original contributions presented in the study are included in the article/Supplementary Material, further inquiries can be directed to the corresponding authors.

\section{ETHICS STATEMENT}

The studies involving human participants were reviewed and approved by Iran National Committee for Ethics in Biomedical Research. Written informed consent for participation was not required for this study in accordance with the national legislation and the institutional requirements.

\section{AUTHOR CONTRIBUTIONS}

EJ: Conceptualization-Equal, Methodology-Equal, Project administration-Equal, Writing-original draft-Equal, Writingreview and editing-Equal; AA: Conceptualization-Equal, Methodology-Equal, Project administration-Equal, Writingoriginal draft-Equal, Writing-review and editing-Equal; NT: Conceptualization-Equal, Methodology-Equal, Project administration-Equal, Writing-original draft-Equal, Writingreview and editing-Equal; AZ: Data curation-Equal, Investigation administration-Equal, Writing-original draftEqual, Writing-review and editing-Equal; FD: Data curationEqual, Investigation-Equal, Writing-original draft-Equal, Writing-review and editing-Equal; AD: Data curation-Equal,

\section{REFERENCES}

Abate, S. M., Ali, S. A., Mantfardo, B., and Basu, B. (2020). Rate of Intensive Care Unit Admission and Outcomes Among Patients with Coronavirus: A Systematic Review and Meta-Analysis. PLoS One. 15, e0235653. doi:10.1371/journal.pone.0235653

An, C., Lim, H., Kim, D.-W., Chang, J. H., Choi, Y. J., and Kim, S. W. (2020). Machine Learning Prediction for Mortality of Patients Diagnosed with COVID19: a Nationwide Korean Cohort Study. Sci. Rep. 10, 1-11. doi:10.1038/s41598020-75767-2

Assaf, D., Gutman, Y. a., Neuman, Y., Segal, G., Amit, S., Gefen-Halevi, S., et al. (2020). Utilization of Machine-Learning Models to Accurately Predict the Risk for Critical COVID-19. Intern. Emerg. Med. 15, 1435-1443. doi:10.1007/ s11739-020-02475-0

Beigel, J. H., Tomashek, K. M., Dodd, L. E., Mehta, A. K., Zingman, B. S., Kalil, A. C., et al. (2020). Remdesivir for the Treatment of Covid-19 - Final Report. N. Engl. J. Med. 383, 1813-1826. doi:10.1056/nejmoa2007764
Investigation Equal, Writing-original draft-Equal, Writingreview and editing-Equal; MB: Data curation-Equal, Investigation-Equal, Writing-original draft-Equal, Writingreview and editing-Equal; HE: Data curation-Equal, Investigation-Equal, Writing-original draft-Equal, Writingreview and editing-Equal; SJ: Data curation-Equal, Investigation-Equal, Writing-original draft-Equal, Writingreview and editing-Equal; SS: Data curation-Equal, Investigation-Equal, Writing-original draft-Equal, Writingreview and editing-Equal; EB: Data curation-Equal, Investigation-Equal, Writing-original draft-Equal, Writingreview and editing-Equal; AM: Data curation-Equal, Investigation-Equal, Writing-original draft-Equal, Writingreview and editing-Equal; AB: Data curation-Equal, Investigation-Equal, Writing-original draft-Equal, Writingreview and editing-Equal; MM: Data curation-Equal, Investigation-Equal, Writing-original draft-Equal, Writingreview and editing-Equal; MS: Data curation-Equal, Investigation-Equal and Writing-original draft-Equal, Writingreview and editing-Equal; MJ: Data curation-Equal, Investigation-Equal and Writing-original draft-Equal; SJ: Conceptualization-Equal, Methodology-Equal, Project administration-Equal, Supervision-Equal, Writing-original draft-Equal, Writing-review and editing-Equal; NM: Conceptualization-Equal, Methodology-Equal, Project administration-Equal, Supervision-Equal, Writing-original draft-Equal, Writing-review and editing-Equal.

\section{FUNDING}

SJ thanks the École polytechnique fédérale de Lausanne for generous support.

\section{SUPPLEMENTARY MATERIAL}

The Supplementary Material for this article can be found online at: https://www.frontiersin.org/articles/10.3389/frai.2021.673527/ full\#supplementary-material

CDC COVID-19 Response Team (2020). Severe Outcomes Among Patients with Coronavirus Disease 2019 (COVID-19) - United States, February 12-March 16, 2020. MMWR. Morb. Mortal. Wkly. Rep. 69(12), 343-346. doi:10.15585/mmwr. $\mathrm{mm} 6912 \mathrm{e} 2$

Collins, G. S., Reitsma, J. B., Altman, D. G., and Moons, K. G. (2015). Transparent Reporting of a Multivariable Prediction Model for Individual Prognosis or Diagnosis (TRIPOD): the TRIPOD Statement. Br. J. Surg. 102, 148-158. doi:10. 1002/bjs. 9736

Emanuel, E. J., Persad, G., Upshur, R., Thome, B., Parker, M., Glickman, A., et al. (2020). Fair Allocation of Scarce Medical Resources in the Time of Covid-19. N. Engl. J. Med. 382, 2049-2055. doi:10.1056/nejmsb2005114

Fumagalli, C., Rozzini, R., Vannini, M., Coccia, F., Cesaroni, G., Mazzeo, F., et al. (2020). Clinical Risk Score to Predict In-Hospital Mortality in COVID-19 Patients: a Retrospective Cohort Study. BMJ Open. 10, e040729. doi:10.1136/ bmjopen-2020-040729

Gao, Y., Cai, G.-Y., Fang, W., Li, H.-Y., Wang, S.-Y., Chen, L., et al. (2020). Machine Learning Based Early Warning System Enables Accurate Mortality Risk 
Prediction for COVID-19. Nat. Commun. 11, 1-10. doi:10.1038/s41467-02018684-2

Garreta, R., and Moncecchi, G. (2013). Learning Scikit-Learn: Machine Learning in Python. Birmingham United Kingdom: Packt Publishing Ltd

Goecks, J., Jalili, V., Heiser, L. M., and Gray, J. W. (2020). How Machine Learning Will Transform Biomedicine. Cell 181, 92-101. doi:10.1016/j.cell.2020.03.022

Goldman, J. D., Lye, D. C. B., Hui, D. S., Marks, K. M., Bruno, R., Montejano, R., et al. (2020). Remdesivir for 5 or 10 Days in Patients with Severe Covid-19. N. Engl. J. Med. 383, 1827-1837. doi:10.1056/nejmoa2015301

Gue, Y. X., Tennyson, M., Gao, J., Ren, S., Kanji, R., and Gorog, D. A. (2020). Development of a Novel Risk Score to Predict Mortality in Patients Admitted to Hospital with COVID-19. Sci. Rep. 10, 1-8. doi:10.1038/s41598-02078505-w

Jamshidi, E., Asgary, A., Tavakoli, N., Zali, A., Esmaily, H., Jamaldini, S. H., et al. (2021a). Using Machine Learning to Predict Mortality for COVID-19 Patients on Day Zero in the ICU. bioRxiv. doi:10.1101/2021.02.04.21251131

Jamshidi, E., Babajani, A., Soltani, P., and Niknejad, H. (2021b). Proposed Mechanisms of Targeting COVID-19 by Delivering Mesenchymal Stem Cells and Their Exosomes to Damaged Organs. Stem Cel Rev Rep 17, 176-192. doi:10.1007/s12015-020-10109-3

Lalmuanawma, S., Hussain, J., and Chhakchhuak, L. (2020). Applications of Machine Learning and Artificial Intelligence for Covid-19 (SARS-CoV-2) Pandemic: A Review. Chaos, Solitons \& Fractals. 139, 110059. doi:10.1016/j. chaos.2020.110059

Levitsky, A., Pernemalm, M., Bernhardson, B.-M., Forshed, J., Kölbeck, K., Olin, M., et al. (2019). Early Symptoms and Sensations as Predictors of Lung Cancer: a Machine Learning Multivariate Model. Sci. Rep. 9, 16504. doi:10.1038/s41598-019-52915-x

Mandrekar, J. N. (2010). Receiver Operating Characteristic Curve in Diagnostic Test Assessment. J. Thorac. Oncol. 5, 1315-1316. doi:10.1097/jto.0b013e3181ec173d

Pan, P., Pan, Y., Xiao, Y., Han, B., Su, L., Su, M., et al. (2020). Prognostic Assessment of COVID-19 in the Intensive Care Unit by Machine Learning Methods: Model Development and Validation. J. Med. Internet Res. 22, e23128. doi:10.2196/23128

Persad, G., Peek, M. E., and Emanuel, E. J. (2020). Fairly Prioritizing Groups for Access to COVID-19 Vaccines. JAMA 324, 1601. doi:10.1001/jama.2020.18513

Snedecor, G. W. (1957). Statistical Methods. Soil Sci. 83, 163. doi:10.1097/ 00010694-195702000-00023
Sun, Q., Qiu, H., Huang, M., and Yang, Y. (2020). Lower Mortality of COVID-19 by Early Recognition and Intervention: Experience from Jiangsu Province. Ann. Intensive Care. 10, 33. doi:10.1186/s13613-020-00650-2

Tabata, S., Imai, K., Kawano, S., Ikeda, M., Kodama, T., Miyoshi, K., et al. (2020). Clinical Characteristics of COVID-19 in 104 People with SARS-CoV-2 Infection on the Diamond Princess Cruise Ship: a Retrospective Analysis. Lancet Infect. Dis. 20, 1043-1050. doi:10.1016/s1473-3099(20)30482-5

Vaid, A., Somani, S., Russak, A. J., De Freitas, J. K., Chaudhry, F. F., Paranjpe, I., et al. (2020). Machine Learning to Predict Mortality and Critical Events in a Cohort of Patients with COVID-19 in New York City: Model Development and Validation. J. Med. Internet Res. 22, e24018. doi:10.2196/24018

Wang, Y., Zhang, D., Du, G., Du, R., Zhao, J., Jin, Y., et al. (2020). Remdesivir in Adults with Severe COVID-19: a Randomised, Double-Blind, PlaceboControlled, Multicentre Trial. The Lancet. 395, 1569-1578. doi:10.1016/ s0140-6736(20)31022-9

WHO (2020). WHO Coronavirus Disease (COVID-19) Dashboard. Available at: https://covid19.who.int (Accessed December 2, 2020)

Wong, T-T. (2015). Performance Evaluation of Classification Algorithms by K-fold and Leave-One-Out Cross Validation. Pattern Recognit. 48, 2839-2846. doi:10. 1016/j.patcog.2015.03.009

Yadaw, A. S., Li, Y. C., Bose, S., Iyengar, R., Bunyavanich, S., and Pandey, G. (2020). Clinical Features of COVID-19 Mortality: Development and Validation of a Clinical Prediction Model. Lancet Digital Health. 2, e516-e525. doi:10.1016/ S2589-7500(20)30217-X

Conflict of Interest: The authors declare that the research was conducted in the absence of any commercial or financial relationships that could be construed as a potential conflict of interest.

Copyright (c) 2021 Jamshidi, Asgary, Tavakoli, Zali, Dastan, Daaee, Badakhshan, Esmaily, Jamaldini, Safari, Bastanhagh, Maher, Babajani, Mehrazi, Sendani Kashi, Jamshidi, Sendani, Rahi and Mansouri. This is an open-access article distributed under the terms of the Creative Commons Attribution License (CC BY). The use, distribution or reproduction in other forums is permitted, provided the original author(s) and the copyright owner(s) are credited and that the original publication in this journal is cited, in accordance with accepted academic practice. No use, distribution or reproduction is permitted which does not comply with these terms. 